\title{
Análise de interações solo-planta-clima em zonas diferenciadas de área de cultivo de milho ${ }^{1}$
}

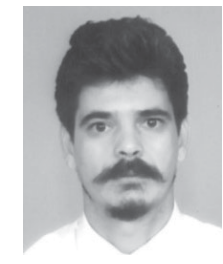

Antonio O. Santos ${ }^{2} \&$ Hélio do Prado ${ }^{3}$

\author{
1 Suporte financeiro FAPESP/PRODETAB \\ ${ }^{2}$ IAC/Centro de Engenharia Agrícola. Rod. Gabriel P.B.Couto, Km 65, CP 26, CEP 13201-970, Jundiaí, SP. Fone: (11) 4582-8155, \\ Fax: (11) 4582-8184. E-mail: odairsan@iac.br (Foto) \\ ${ }^{3}$ IAC/Centro de Cana-de-açúcar. Rod. Anel Viário Km 221, CP 221, CEP 14001-970, Ribeirão Preto, SP. Fone: (16) 637-2650 \\ Protocolo $161-14 / 11 / 2001$
}

\begin{abstract}
Resumo: A expressiva área de cultivo de milho no Brasil e a necessidade constante de aprimoramento do seu manejo, justificam o estudo sobre novas metodologias de tratamento dos recursos financeiros ou técnicos destinados à produção. A cultura do milho tem demonstrado significativa variabilidade espacial da produtividade em estudos sobre a questão e variabilidade temporal também pode ser esperada, ao longo das estações de crescimento. A observação do crescimento e do desenvolvimento de cultivos através de modelos de simulação, pode servir de apoio para o estudo das causas das variabilidades citadas, além de atuar como elemento de integração de dados de solo, planta e clima, principalmente em estudos sobre implementação do manejo localizado, onde se busca aproveitar diferenças de potencial produtivo na mesma área de cultivo. Em uma área de produção de milho do município de Angatuba, SP, região do Vale do Paranapanema $\left(23^{\circ} 33^{\prime} \mathrm{S} ; 48^{\circ} 18^{\prime} \mathrm{W} ; 670 \mathrm{~m}\right)$, foram levantados dados de solo, planta e clima, adequados ao banco de dados do modelo Ceres-Maize, para simulações de elementos envolvidos na produtividade da cultura de milho, em uma estação de crescimento e ao longo de uma série climática de médio prazo, simulando-se, separadamente, para três tipos de solo classificados na área. O modelo Ceres-Maize possibilita a simulação do crescimento e desenvolvimento da cultura do milho, em escala diária, com base no balanço de carbono, nitrogênio e água, para uma cultivar com características morfogenéticas conhecidas. Interações de solo, planta e clima, causaram diferenças moderadas nos padrões de flutuação da produtividade simulada ao longo dos anos, em duas zonas distintas, dentro da mesma área de cultivo de milho, diferenciando moderadamente as dosagens de nitrogênio para o máximo rendimento econômico. A simulação evidenciou que a aplicação do modelo como ferramenta de análise "não pontual" está condicionada ao contraste espacial existente nas propriedades físicas do solo e no potencial produtivo dos cultivos.
\end{abstract}

Palavras-chave: Ceres-Maize, agrometeorologia, água e solo, Zea mays L.

\section{Site-specific analysis of soil-plant-climate interactions on maize}

\begin{abstract}
Taking into account the very large area of corn crop in Brazil along side the constant demand for the improvement of crop management, there is a need for further investigation into the new methodologies for the treatment of inputs required by the production system. Maize yield studies have demonstrated significant spatial and time-related variability along the growing seasons. The simulation of the crop development may serve while discussing the variability and its effects on yield, as well as for data integration, when looking for useful information. This happens, mainly when moving from a homogeneous treatment of cultivated field toward a site-specific based management; where the difference in potential yield is due to occur, inside the same cultivated area. In accordance with the Ceres-Maize model, soil, plant and climate data, were collected in a corn growing area, located around the Angatuba (Sao Paulo), Brazil, at the Vale do Parapanema region (2333' S; $48^{\circ} 18^{\prime} \mathrm{W} ; 670 \mathrm{~m}$ ). The simulation of yield related properties, for a single growing season and for a medium term climatic series of data was performed, in three separate soil units. The Ceres-Maize model allows the simulation of corn development on a daily-step basis, based upon a balance of carbon, nitrogen and water, provided that key morphogenetic traits of a given cultivar are known. Soil, plant and climate interactions implied moderate differences in the pattern of simulated corn yield variability along years, for two separate zones, inside the same cultivated area. This caused the simulated rate of nitrogen for the maximum economic return, to be moderately different. The simulation process suggested that the use of the model as a "non-point" analytical tool is conditional to the level of spatial contrasts in soil physical properties and yield potential.
\end{abstract}

Key words: Ceres-Maize, agrometeorology, soil-water, Zea mays L. 


\section{INTRODUÇÃO}

O milho está entre as graníferas de alta importância na produção agrícola do Brasil, haja vista a significativa área cultivada e a destinação, tanto para consumo humano como animal. Por outro lado, esta cultura pode apresentar alta variabilidade temporal da produtividade, causada principalmente por variações em elementos climáticos (Bergonci et al., 2001). Variação espacial significativa da produtividade, dentro de um mesmo ano e área, foi relatada por Santos et al. (2000).

Estes aspectos, justificam o estudo continuado sobre o aprimoramento de técnicas de manejo da referida cultura, sobretudo em face da adoção de novos métodos, através dos quais se busca aproveitar a variabilidade espacial e temporal da produtividade, como subsídio informativo, para uma abordagem diferencial do potencial produtivo da cultura, em uma mesma área (Plant, 2001).

De fato, a discussão sobre este tema, que se enquadra no conjunto de técnicas denominado agricultura de precisão, tem crescido de importância, ainda que de forma acadêmica. A implementação deste tipo de tecnologia tem sido abordada com base em estudos feitos em áreas de pequena extensão e em amostragem intensiva do meio físico. Para áreas maiores, aquela implementação pode ser dificultada, pelo grande número de dados que podem ser gerados, a partir de amostragem intensiva; no entanto, uma escala realística de trabalho, condizente com a viabilidade técnica e econômica da amostragem e a utilização de sensoriamento remoto, podem ajudar a viabilizar a implementação discutida (Moran et al., 1997; Santos et al., 2001a). Por outro lado, no ambiente discutido a simulação e a modelagem de processos físicos, relacionados com a produtividade final de cultivos agrícolas, apresentam-se como uma escolha natural, face à necessidade de integração de grande número de dados e conseqüente geração de informação útil.

Devido aos altos custos e ao tempo geralmente envolvidos na experimentação agrícola, a utilização da simulação do crescimento e do desenvolvimento apresenta-se como parte da solução, quando se buscam resultados de pesquisa em qualquer ambiente de estudo (Faria et al., 1997).

A modelagem dos processos envolvidos na produção agrícola tem sido largamente testada como ferramenta de suporte à decisão em áreas de cultivo. O modelo Ceres-Maize (Jones \& Kiniry, 1986), incorporado à interface gráfico-numérica DSSAT3.5 (Tsuji et al., 1994) possibilita a simulação semiempírica do crescimento e desenvolvimento da cultura do milho, em escala diária, com base no balanço de carbono, nitrogênio e água. Como resultado, pode-se obter, entre outros parâmetros, a duração dos estágios fenológicos, acumulação de biomassa, produção de grãos, para diferentes tipos de solo, clima e condições de manejo, para uma cultivar com características morfogenéticas conhecidas (coeficientes genéticos).

O modelo Ceres-Maize opera com um balanço hídrico que simula o fluxo e a absorção da água para cada camada estabelecida do horizonte do solo. A redistribuição da água no perfil do solo tem, como base, um sistema em cascata, cujos fluxos saturado e não-saturado, podem ocorrer entre camadas adjacentes, baseados na diferença do conteúdo da água volumétrica entre camadas (Johnson \& Ritchie, 1989).
A dinâmica do nitrogênio está incorporada a sub-rotinas que simulam a mineralização da matéria orgânica, com conseqüente imobilização de $\mathrm{N}$, nitrificação/denitrificação de amônio e hidrólise da uréia, levando-se em consideração informações de entrada, como teores de carbono orgânico, $\mathrm{pH}$, densidade do solo e dados meteorológicos (Godwin \& Jones, 1991).

O modelo Ceres-Maize tem sido testado em muitos locais ao redor do globo e a sua aplicabilidade verificada em várias condições ambientais, desde climas tropicais até temperados (Keating et al., 1991; Jones et al., 1998). Sua aplicação pode ser feita tanto para escala de campo como de parcela (Jones \& Kiniry, 1986). Portanto, tendo em vista a necessidade de se observar mais a fundo o ambiente interno de instalação de uma cultura de milho, uma análise exploratória pode ser efetuada para contribuir na discussão sobre simulação aplicada à cultura. Igualmente, pode-se verificar o potencial existente para um manejo diferenciado, na mesma área, colocando-se em perspectiva a dualidade "manejo homogêneo versus manejo localizado".

Objetivou-se, com este trabalho, testar o modelo CeresMaize em escala inferior à de uma área cultivada e modelar alguns elementos da produção, para uma estação de crescimento e para um período climático de médio prazo.

\section{MATERIAL E MÉTODOS}

\section{Local, época e solo}

O experimento foi realizado em Angatuba, SP (233' S; $48^{\circ} 18^{\prime} \mathrm{W} ; 670 \mathrm{~m}$ ) para o qual se utilizou uma área de milho irrigado de 34,3 ha. A área está estabelecida sob sistema de plantio semi-direto, com a rotação trigo/aveia e milho/feijão, com interrupção a cada 4 - 5 anos para instalação de cultivo de batata.

A cultivar híbrido Pioneer 30F33 foi instalada nos anos agrícolas 1999/2000 e 2000/2001, obedecendo a programação específica do proprietário da área, na qual foi demarcada uma malha quadrada de 33 pontos, eqüidistantes em $100 \mathrm{~m}$ e, em cada ponto, foram levantados dados de física e fertilidade do solo, nas profundidades de 0 - 20, 20 - 40, 40 - 60 e $60-100 \mathrm{~cm}$. As amostras de solo foram coletadas com amostrador dinâmico de acionamento hidráulico.

O levantamento ultra-detalhado de elementos de pedologia, feito na mesma área, permitiu a classificação do solo, em três classes predominantes. Latossolo Vermelho distrófico típico, textura argilosa, A moderado (LVd-2), Latossolo Vermelho amarelo distrófico típico, textura muito argilosa, A moderado (LVAd) e Latossolo Vermelho distrófico típico álico, textura muito argilosa, A moderado (LVd-1).

\section{Dados da cultura e do clima}

A cultivar de milho, híbrido Pioneer 30F33, foi instalada em ambos os anos experimentais, na segunda quinzena de setembro, observando-se a recomendação da pesquisa regional, para alto rendimento sob irrigação (Raij et al., 1997).

A irrigação do milho foi feita através do sistema "pivôcentral". Uma lâmina total em torno de $225 \mathrm{~mm}$ foi aplicada durante cada estação de crescimento da cultura (1999/2000 e 2000/2001), distribuída em aplicações esporádicas. Um teste específico foi efetuado na área para verificação da quantidade 
e da uniformidade de distribuição da lâmina de irrigação, tendo-se observado coerência nos parâmetros de calibração do fabricante do sistema de irrigação, tanto para a lâmina quanto para uniformidade de distribuição da água.

Os dados meteorológicos, entre eles radiação solar global, velocidade do vento, temperatura máxima e mínima e precipitação pluviométrica, foram obtidos em estação meteorológica instalada ao lado da área experimental. Além disso, um período de 10 anos de dados meteorológicos foi utilizado para simulação de médio prazo. Para esta simulação seriada, coletaram-se dados de precipitação pluviométrica em posto do Departamento de Águas e Energia Elétrica (DAEE-SP), localizado a $1000 \mathrm{~m}$ de distância do experimento. Outros dados meteorológicos foram obtidos em estação de coleta do Instituto Agronômico (IAC) em Tatuí (SP), município vizinho ao experimento.

O milho esteve sob observação durante duas estações de crescimento (1999/2000 e 2000/2001) quando fases fenológicas e alguns componentes do rendimento foram acompanhados, a fim de se obter a calibração de coeficientes genéticos, notadamente a ocorrência temporal da emergência, período juvenil, florescimento, maturação fisiológica e rendimento final.

A unidade de solo LVd-1 representa a quase totalidade da metade oeste da área experimental. Nesta área, o manejo da cobertura de inverno (aveia) foi feito de maneira diferenciada. A cobertura verde foi removida para fenação, nas duas estações de crescimento em observação. Em função disto, as estimativas de resíduo remanescente (parte aérea e raiz), assim como nitrogênio inicial (Tsuji et al., 1994), necessários à simulação, foram tratados diferentemente, em relação às unidades de solo LAVd e LVd-2.

\section{Simulação}

O modelo Ceres-Maize, incorporado à interface gráfica DSSAT3.5 (IBSNAT, 1990) foi utilizado em cada tipo de solo, na área experimental, e a verificação de contrastes foi efetuada para elementos do balanço hídrico e dinâmica de nitrogênio. Este modelo, que simula o crescimento e o desenvolvimento em marcha diária, é sensível a variações nas condições de solo, água, clima e manejo. A produção potencial de matéria seca é uma função da radiação fotossinteticamente ativa, sendo o acúmulo diário particionado entre órgãos da planta em crescimento ativo no período considerado. $\mathrm{O}$ cumprimento das fases fenológicas está associado à acumulação térmica, calculada em graus-dia. Portanto, o funcionamento do modelo depende principalmente do banco de dados de solo, clima e manejo, que se inter-relacionam.

A verificação da interação temporal solo-planta-clima foi efetuada através de uma simulação "ex-post", de médio prazo, utilizando-se dados meteorológicos referentes a 10 estações de crescimento (1988 a 1998). Os anos de observação constituem uma amostra razoável do histórico climático da região de cultivo. Nesta simulação, seis dosagens de nitrogênio $(0,20$, $50,100,150$ e $200 \mathrm{~kg} \mathrm{ha}^{-1}$ ) foram testadas e a flutuação do rendimento observada.

Em função de dosagens crescentes de nitrogênio (1/3 na base e $2 / 3$ em cobertura, aos $30 \mathrm{~d}$ após a semeadura) o retorno econômico (bruto) do cultivo do milho para cada tipo de solo classificado na área, foi simulado para a série de 10 estações de crescimento, a qual teve, por base, a seguinte formulação simples:

$$
\mathrm{MR}=\left(\mathrm{PP}_{\mathrm{r}}\right)-\left(\mathrm{NP}_{\mathrm{n}}\right)
$$

em que MR é o máximo rendimento econômico, $\mathrm{P}$ é a produtividade do milho $\left(t h^{-1}\right), P_{r}$ é o preço de venda $\left(R \$ t^{-1}\right)$, N é a dosagem de nitrogênio $\left(\mathrm{kg} \mathrm{ha}^{-1}\right)$ e $P_{n}$ é o preço do nitrogênio $\left(\mathrm{R} \$ \mathrm{~kg}^{-1}\right)$. Outros custos fixos e variáveis não foram considerados, por serem os três tipos de solo um só sistema, sob o ponto de vista de aplicação de recursos. Assim, uma otimização para dosagens de nitrogênio foi efetuada, considerando-se os três tipos de solo, as condições de manejo e a incidência diária do microclima.

\section{RESULTADOS E DISCUSSÃO}

A Figura 1 indica a distribuição dos solos classificados na área, para os quais se procedeu, separadamente, a simulação para parâmetros relacionados com o rendimento final. A ocorrência de tipos de solo caracterizou-se pela predominância de duas unidades principais, LVd-1 e LVd-2, que ocupam a maior parte da área.

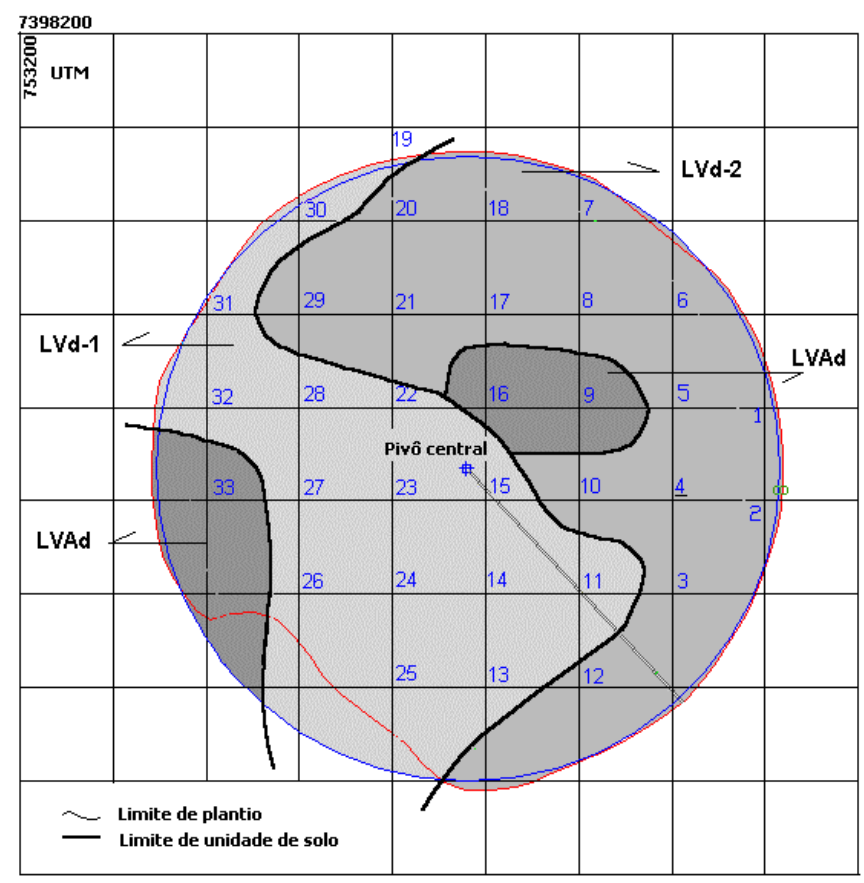

Figura 1. Distribuição de malha amostral e dos tipos de solo classificados em área de 34,3 ha cultivados com milho, nas estações de crescimento 1999/2000 e 2000/2001, em Angatuba, SP

Partindo-se dos princípios da agricultura de precisão, a classificação de tipos diferentes de solo em uma área pequena sugere oportunidades de manejo diferenciado. Muito embora, tipo de solo e produtividade não tenham um relacionamento direto, a ocorrência deste fato em áreas maiores, aponta um caminho promissor para a aplicação da simulação e modelagem, "não-pontual", principalmente quando na obtenção dos dados, de solo e planta, se incluem fontes relacionadas ao sensoriamento remoto, para o estudo de contrastes do meio físico.

As propriedades físico-químicas das unidades de solo classificadas na área experimental estão mostrados na Tabela 1 , onde se notam diferenças quanto aos perfis estudados, 
Tabela 1. Propriedades físicas e de fertilidade, para as unidades de solo LVd-2, LVAd e LVd-1 componentes do arquivo Ceres-Maize, para simulação do crescimento e desenvolvimento, em lavoura de milho, em Angatuba, SP (IBSNAT, 1990)

\begin{tabular}{|c|c|c|c|c|c|c|c|c|c|c|}
\hline \multirow{2}{*}{$\begin{array}{c}\text { Camada } \\
\text { do Solo } \\
\mathrm{cm}\end{array}$} & \multirow{2}{*}{$\begin{array}{c}\text { Argila } \\
\varnothing<0,002 \mathrm{~mm}\end{array}$} & \multirow{2}{*}{$\begin{array}{c}\text { Silte } \\
0,053<\varnothing<0,002 \mathrm{~mm}\end{array}$} & $\theta-1500 \mathrm{kPa}$ & $\theta-33 \mathrm{kPa}$ & $\theta$-Sat & \multirow{2}{*}{$\begin{array}{l}\mathrm{K} \text { sat } \\
\mathrm{cm} \mathrm{h}^{-1}\end{array}$} & \multirow{2}{*}{$\begin{array}{c}\text { Ds } \\
\mathrm{g} \mathrm{cm}^{-3}\end{array}$} & \multirow{2}{*}{$\begin{array}{c}\mathrm{pH} \\
\text { água }\end{array}$} & \multirow{2}{*}{$\begin{array}{c}\mathrm{C} \\
\%\end{array}$} & \multirow{2}{*}{$\begin{array}{c}\text { CTC } \\
\mathrm{cmol}_{\mathrm{c}} \mathrm{kg}^{-1}\end{array}$} \\
\hline & & & \multicolumn{2}{|c|}{$\mathrm{cm}^{3} \mathrm{~cm}^{-3}$} & & & & & & \\
\hline \multicolumn{11}{|c|}{ Latossolo Vermelho distrófico típico textura argilosa - LVd-2 } \\
\hline $0-20$ & 60,20 & 15,10 & 0,191 & 0,349 & 0,579 & 3,78 & 0,99 & 5,0 & 2,03 & 9,53 \\
\hline $20-40$ & 59,00 & 13,12 & 0,190 & 0,329 & 0,536 & 3,06 & 0,99 & 4,9 & 1,80 & 9,00 \\
\hline $40-65$ & 60,10 & 12,15 & 0,200 & 0,329 & 0,643 & 3,91 & 0,97 & 4,3 & 1,04 & 8,21 \\
\hline $65-100$ & 59,12 & 12,45 & 0,109 & 0,320 & 0,575 & 1,54 & 1,05 & 4,2 & 1,04 & 8,21 \\
\hline \multicolumn{11}{|c|}{ Latossolo Vermelho distrófico típico textura muito argilosa - LVAd } \\
\hline $0-20$ & 67,25 & 15,78 & 0,250 & 0,376 & 0,635 & 3,32 & 1,02 & 4,8 & 2,68 & 9,89 \\
\hline $20-40$ & 66,92 & 13,45 & 0,250 & 0,408 & 0,612 & 2,86 & 0,99 & 4,4 & 2,35 & 9,52 \\
\hline $40-65$ & 65,44 & 18,31 & 0,278 & 0,409 & 0,612 & 1,98 & 0,97 & 4,2 & 1,27 & 7,17 \\
\hline $65-100$ & 64,96 & 12,05 & 0,270 & 0,390 & 0,595 & 1,31 & 1,16 & 4,3 & 1,21 & 7,49 \\
\hline \multicolumn{11}{|c|}{ Latossolo Vermelho distrófico típico álico - LVd-1 } \\
\hline $0-20$ & 66,63 & 15,09 & 0,225 & 0,366 & 0,59 & 3,70 & 1,10 & 4,7 & 1,81 & 9,56 \\
\hline $20-40$ & 64,47 & 13,55 & 0,271 & 0,410 & 0,60 & 1,74 & 1,23 & 4,3 & 1,45 & 8,62 \\
\hline $40-65$ & 66,66 & 13,90 & 0,276 & 0,393 & 0,65 & 2,94 & 1,04 & 4,1 & 1,20 & 8,00 \\
\hline $65-100$ & 65,40 & 15,15 & 0,270 & 0,407 & 0,59 & 1,47 & 1,28 & 4,1 & 1,03 & 8,00 \\
\hline
\end{tabular}

$\theta$ - umidade do solo; sat - saturação; $\mathrm{k}$ - condutividade hidráulica; Ds - densidade do solo; $\varnothing$ - diâmetro das partículas do solo e C - carbono orgânico

notadamente nos parâmetros relacionados à retenção e movimento da água no solo.

A simulação em uma estação de crescimento evidenciou contrastes na fração da água extraível, ao longo do ciclo da cultura do milho (Fig. 2). Este fato está ligado à variabilidade de propriedades físicas observadas na área experimental. A água extraível, por sua vez, representa a faixa empírica limitada pela capacidade de campo (33 $\mathrm{kPa}$ ) e o ponto de murcha permanente $(1500 \mathrm{kPa})$, interagindo com a incidência de lâminas de recargas, ou seja, lâminas da irrigação e provenientes da precipitação pluvial.

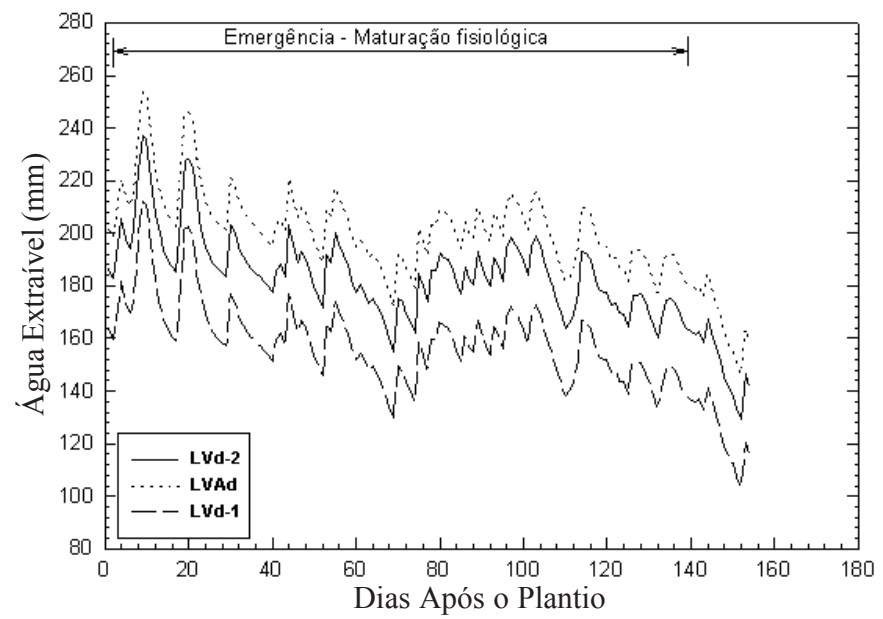

Figura 2. Água extraível, ao longo do ciclo do milho, para três tipos de solos, na estação de crescimento 1999/2000

Muito embora não estejam sendo considerados outros elementos do balanço hídrico, como drenagem profunda ou ascensão capilar, por exemplo, pode-se deduzir que as unidades de solo LVAd e LVd-2 apresentam um potencial maior de água disponível ao longo do ciclo do milho. Por outro lado, um acompanhamento melhor do balanço hídrico diário e as ocorrências das fases do milho, nessas três unidades de solo, seriam necessários para demonstrar se uma fração maior de água potencial disponível estaria relacionada com maior disponibilidade de água efetiva. Rosa et al. (2001) em estudo sobre esta questão, demonstraram, através do acompanhamento do IAF e da água no solo, em solos de texturas diferentes, que nem sempre uma quantidade maior de água armazenada no solo está ligada diretamente a uma disponibilidade hídrica maior.

Devido ao fato de que o potencial de lixiviação de nitrogênio está ligado diretamente ao movimento da água no solo depreende-se, a partir da Figura 2, que há diferenças entre os solos estudados quanto ao potencial de lixiviação de nitrogênio. O mesmo raciocínio pode ser feito quanto ao potencial de movimentação de agroquímicos no perfil dos solos. Este fato tem particular importância porque a área em questão recebe o cultivo da batata em períodos de rotação, sendo que seu cultivo é caracterizado por adubações e aplicações de defensivos agrícolas em grande intensidade, com impactos ambientais importantes. Neste caso, o manejo da água, da adubação e agroquímicos, teriam influências diferentes para os três tipos de solo estudados, quanto ao potencial para contaminação do lençol freático.

A exploração de interações de solo, clima e desenvolvimento de cultivos, pode ser cotejada ao longo dos anos. Com isto, o fenômeno da produtividade pode ser observado frente à ocorrência de diferentes condições de disponibilidade hídrica e flutuações, na incidência das variáveis meteorológicas, numa marcha diária. Este aspecto da simulação do crescimento e do desenvolvimento de cultivos permite a obtenção de uma análise "ex-ante" do comportamento vegetal, o que substitui com propriedade a necessidade de experimentação a campo.

A Figura 3 exibe os resultados de simulação obtidos através do modelo Ceres-Maize, para dez estações de crescimento, para os dois tipos de solos mais contrastantes da área experimental, em termos de propriedades físicas e manejo dispensado.

Na Figura 3, a comparação dos resultados obtidos na simulação, para toda a série de anos, nos dois tipos de solo mostrados, permite verificar-se que os valores de produtividade, para o primeiro ano, são relativamente similares para as dosagens mais altas de nitrogênio. Nas dosagens menores ocorre maior diferença, devido, provavelmente, às condições de solo e do manejo. Com o passar dos anos ocorre flutuação diferenciada 

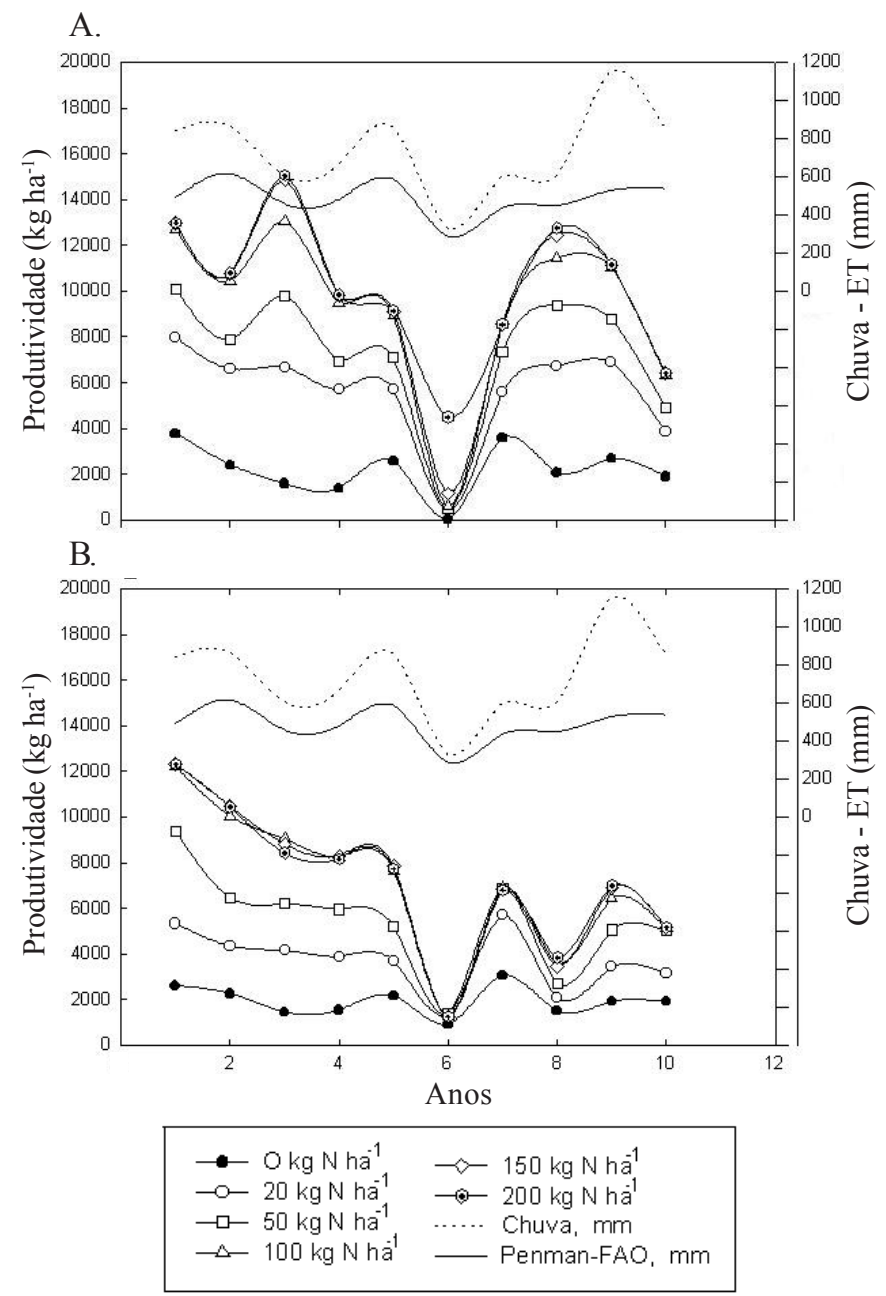

Figura 3. Produtividade simulada de milho irrigado para seis dosagens de nitrogênio, ao longo de dez estações de crescimento, em Angatuba, SP, para duas unidades de solo; (A) LVd-2 e (B) LVd-1

para o relacionamento entre produtividade e dosagens de nitrogênio, o que evidencia o impacto da interação clima e condições de planta e solo. Para o ano em que ocorre a maior restrição pluviométrica, observa-se o nivelamento em baixos valores, nas duas unidades de solo mostradas.

A flutuação temporal diferencial da produtividade, para os dois tipos de solo e na mesma área de cultivo, tem particular importância, por evidenciar a variabilidade temporal da interação simulada no presente estudo. A flutuação observada teria, provavelmente, o poder de confundir a variabilidade espacial que possa ocorrer dentro de uma mesma área sugerindo-se, portanto, a partir da Figura 3, que um padrão de distribuição espacial da produtividade, governado por condições do manejo anual, pode vir a ser mascarado ao logo dos anos, pelo fator climático, o que impõe, ao fenômeno da distribuição espacial da produtividade em uma área de cultivo, um padrão de complexidade que somente poderia ser tratado dentro de um programa de manejo diferenciado, se um contraste forte entre os elementos mais diretamente ligados à produtividade, existir dentro da lavoura, conforme discutiram Santos et al. (2001b).

$\mathrm{Na}$ Figura 4 tem-se o resultado da simulação ao longo da série de dez estações de crescimento, para a relação entre máximo rendimento econômico e dosagens crescentes de nitrogênio.

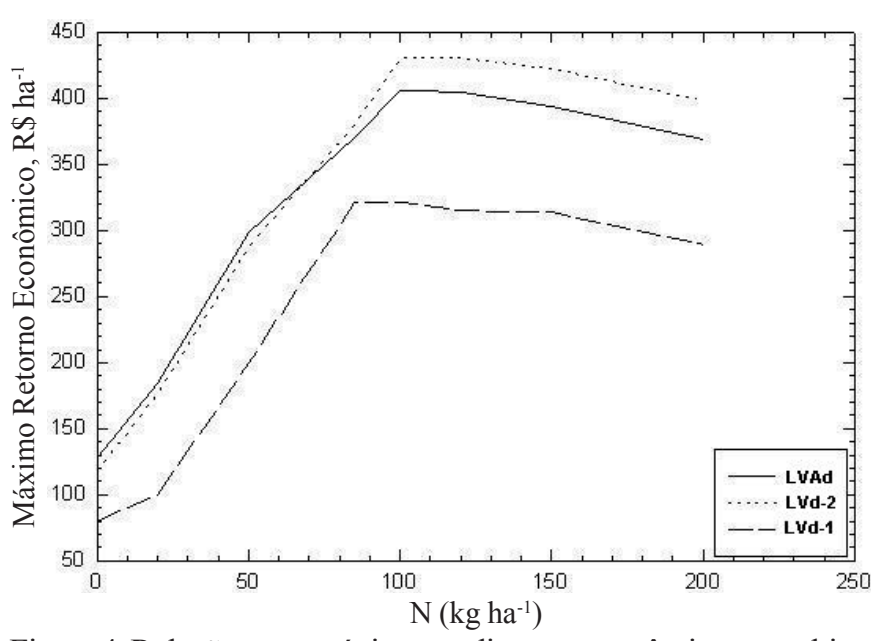

Figura 4. Relação entre máximo rendimento econômico em cultivo de milho e dosagens de nitrogênio, simuladas para uma série de 10 anos de dados meteorológicos, para três tipos de solo diferentes

Esses resultados estão de acordo com o que vem sendo praticado na área, em termos de dosagem de adubação nitrogenada, evidenciando que a otimização apresentada é realística. Nota-se, no entanto, uma pequena diferença entre LVd-2 e LVAd no ponto ótimo de nitrogênio. Por outro lado, LVd-1 diferenciou-se em maior grau dos outros dois tipos de solo, apresentando um pico de máximo rendimento econômico, abaixo de $90 \mathrm{~kg} \mathrm{ha}^{-1}$. Esses resultados evidenciam o impacto da variabilidade climática sobre a produção, associado às diferenças nas características de solo, dentro da mesma área. Este fato, analisado conjuntamente com o potencial de lixiviação de $\mathrm{N}$, já discutido, além dos aspectos de disponibilidade hídrica observados na Figura 2 sugere que, dentro de uma mesma área, podem ocorrer potenciais produtivos diferenciados, que poderiam justificar estratégias de manejo em separado, para alguns recursos da produção.

Muito embora o nível de variabilidade dos principais elementos de solo, impactantes da produtividade, não ocorram nesta área com grande contraste, é possível antever que a simulação e a modelagem constituem, nos moldes deste trabalho, um meio eficaz para o integração de dados neste contexto, principalmente para áreas maiores onde grandes contrastes podem eventualmente ocorrer e, neste caso, haveria necessidade dos procedimentos de simulação aqui discutidos, como forma de se aproveitar a variabilidade de propriedades de solo e planta, para o manejo diferenciado da lavoura, quando fossem buscados, principalmente a otimização de recursos. Este fato tem particular importância quando se objetiva desenvolver sistemas de suporte à decisão, com base em modelos de simulação, ligados em Sistema de Informação Geográfica (SIG) sobretudo quando os contrastes espaciais de elementos condicionantes da produtividade permitem um delineamento claro de zonas de manejo nas quais existe uma bem definida diferenciação de potencial produtivo, na mesma área de cultivo. Neste caso, mais claramente se justificariam práticas de manejo diferenciado (Jones \& Barnes, 2000). Além disto, trabalhos neste campo requerem um número de dados não factíveis por amostragem e experimentação a campo, tendo-se como alternativa, a simulação nos moldes deste trabalho. Por outro 
lado e dado ao nível de complexidade e variabilidade dos parâmetros que envolvem a produtividade dos cultivos, outros elementos diretamente relacionados com a produtividade, necessitariam ser melhor equacionados e incorporados ao tipo de simulação discutida, principalmente a variação da temperatura do solo, a resistência à penetração de raízes e a aeração do solo.

\section{CONCLUSÕES}

A partir das observações feitas na área experimental conclui-se que:

1. A simulação diferenciada (não-pontual), com base na fragmentação de uma área de cultivo, é tanto mais aplicável, quanto mais os contrastes espaciais de propriedades físicas e do potencial produtivo dos cultivos forem evidentes.

2. Interações temporais de solo, planta e clima podem influenciar a expressão espacial conjunta da produtividade e da resposta à dosagens de nitrogênio, em uma mesma área de cultivo.

\section{LITERATURA CITADA}

Bergonci, J.I.; Bergamaschi, J.I.; Santos, A.O.; França, S.; Radin, B. Eficiência da irrigação em rendimento de grãos e matéria seca de milho. Pesquisa Agropecuária Brasileira, Brasília, v.36, n.7, p.949-956, 2001.

Faria, R.T.; Folegatti, M.V.; Frizzone, J.A.; Saad, A.M. Determination of a long-term optimal irrigation strategy for dry beans in Parana State, Brazil. Scientia Agricola, Piracicaba, Número especial, p.155-164, 1997.

Godwin, D.C.; Jones, C.A. Nitrogen dynamics in soil-plant system. In: Hanks, R.J.; Ritchie, J.T. (eds.). Modelling plant and soil system. Madison: ASA, CSSA, SSSA, 1991. n.38, p.86-112. Monogafia

IBSNAT - International Benchmark Site Network for Agrotechnology Transfer. Progress Report 1987-1990. Honolulu, 1990.54p.

Johnson, B.S.; Ritchie, J.T. Simulation of soil water balance in IBSNAT crop models. In: IBSNAT Symposium, 1989, Michigan. Anais... Michigan: Michigan State University, 1989. v.1, p.249-255.

Jones, D.; Barnes, E.M. Fuzzy composite programming to combine remote sensing and crop models for decision support in precision crop management. Agricultural System, Amsterdam, v.65, p.137-158, 2000.
Jones, J.W.; Kiniry, J.R. Ceres-Maize. A simulation model of maize growth and development. College Station: Texas A\&M University Press, 1986. 56p.

Jones, J.W.; Tsuji, G.Y.; Hoogenboom, G. Decision support system for agrotechnology transfer: DSSAT v3. In: Tsuji, G.Y., Hoogenboom, G., Thornton, P.K. (eds) Understanding options for agricultural production. Dordrecht: Kluwer Academic Publishers. 1998. p.157-177.

Keating, B.A.; Godwin, D.C.; Watiki, J.M. Optimising nitrogen inputs in response to climatic risk. In: Muchow, R.C.; Bellamy, J.A.(eds.). Climatic risk in crop production: Models and management for the semiarid tropics and subtropics. Wallingford: Oxon, 1991.38p.

Moran, M.S.; Inoue, Y.; Barnes, E.M. Opportunities and limitations for image-based remote sensing in precision crop management. Remote Sensing and Environment, Amsterdam, v.61, p.319-346, 1997.

Plant, R.E. Site-specific management: The application of information technology to crop production. Computers and Eletronics in Agriculture, Amesterdam, v.30, p.9-29, 2001.

Raij, B.V.; Cantarella, H. Quaggio, J.A.; Furlani, A.M.C. Recomendações de adubação e calagem para o Estado de São Paulo. Campinas: Instituto Agronômico, 1997. 285p. Boletim Técnico, 100

Rosa, G.M.; Carlesso, R.; Petry, M.T. Variação do armazenamento de água em solos de diferentes texturas para a cultura do feijoeiro irrigado e submetido a déficit hídrico. In: Congresso Brasileiro de Agrometeorologia, 12, 2001, Fortaleza. Anais...Fortaleza: Sociedade Brasileira de Agrometeorologia, 2001. v.1, p.523-524.

Santos, A.O.; Maziero, J.V.G.; Cavalli, A.C.; Moraes, J.F.L.; Yanai, K. Monitoramento localizado da produtividade de milho cultivado sob irrigação. Revista Brasileira de Engenharia Agrícola e Ambiental, Campina Grande, v.5, p.88-95, 2001a.

Santos, A.O.; Maziero, J.V.G.; Valeriano, M.M. Cavalli, A.C.; Correa, I.M.; Moraes, J.F.L.; Rosa-Filho, G. Variabilidade da produtividade de milho irrigado, um estudo de caso em São Paulo, Brasil. In: Congreso Latinoamericano y del Caribe de Ingenieria Agrícola, 10, 2000. Anais... Irapuato: Associação Latinoamericana y del Caribe de Ingenieria Agrícola, v.1, 2000, CD-Rom

Santos, A.O.; Pallone-Filho, W.J.; Cavalli, A.C. Contrastes espaciais de variáveis da água no solo obtidos por medição e modelagem. In: Congresso Brasileiro de Agrometeorologia, 12, 2000, Fortaleza. Anais...Fortaleza: Sociedade Brasileira de Agrometeorologia, 2001b. v.1, p.383-385.

Tsuji, G.Y.; Uehara, G.; Balas, S. DSSAT Version 3, Vol. 2. Honolulu: International Benchmark Sites Network for Agrotechnology Transfer, 1994. 500p. 